\title{
Morphological Evolution and Junction Dynamics at Faceted Grain Boundaries
}

\author{
D.L. Medlin
}

Thin Film and Interface Science Department, Organization 8721, Mail Stop 9161, Sandia National Laboratories, Livermore, CA 94551

Grain boundaries are often faceted in extreme cases of interfacial anisotropy. Such a morphology prevents the application of classical notions of grain-boundary curvature to understanding microstructural evolution. Although there has been much effort at incorporating anisotropic grainboundary properties, including faceted geometries, into computational approaches for microstructural evolution, at present our mechanistic understanding of the behavior of facets and their junctions remains limited.

We are employing in situ transmission electron microscopy to better understand the behavior of facets, and their junctions, during grain evolution. In this presentation we consider the dynamics of $\Sigma=3\{11 \overline{2}\}$ facets in $<111>$ oriented gold thin films. This system is ideally suited for such studies since the crystallography and structure of the boundaries is already well understood [e.g., 1]. An example of one such faceted grain is shown in Figure 1a to illustrate the fine-scale facet distribution typical of the initial, as-deposited films. As shown in Figure 1b, annealing dramatically increases the fraction of the boundary length that is composed of larger-scale facets. Classical, capillaritybased models for facet evolution, which neglect the energetics and interactions of the junctions between facets, predict that such boundaries should evolve only through the motion of non-perimeter conserving facets (i.e., facets terminated by corners of the same sense with respect to the Wulff surface). However, by tracking the real-time evolution of such boundaries using in situ TEM, we have shown that perimeter-conserving facets (i.e., with corners of opposite sense) play a significant role [2]. An example showing the transition from an initial highly stepped boundary to a single corner is shown in the series of micrographs in Figure 2. These results point to the need for an improved understanding of the properties of the facet junctions.

That these junctions might have more than just geometrical significance is not necessarily surprising. For instance, Hetherington et al., have identified a dislocation-like strain field at $\mathrm{Au}\{11 \overline{2}\}$ junctions that arises from the incompatibility of the interfacial translation vectors of the adjacent boundary segments [1]. In situ measurements provide a means of probing the interactions between the junctions. An analysis of the thermal fluctuations in junction separation (Figure 3), measured from digitized, real-time video data, shows a departure from random-walk behavior--an important signature of junction-junction interactions. Methods for quantifying such measurements and comparisons with models for junction-interaction will be discussed.

\section{References}

[1] C.J.D. Hetherington, U.Dahmen, J.-M. Pénisson, in "Atomic Resolution Microscopy of Surfaces and Interfaces," ed. D.J. Smith (MRS Proceedings, Vol. 466, 1997) 215-226.

[2] D.L. Medlin and G. Lucadamo, in "Influences of Interface and Dislocation Behavior on Microstructure Evolution," eds. M. Aindow et al. (MRS Proceedings, Vol 652, 2001) 3.4.1-6.

[3] This research is supported by the U.S. Department of Energy, in part by the Office of Basic Energy Sciences, under contract number DE-AC04-94-AL85000 

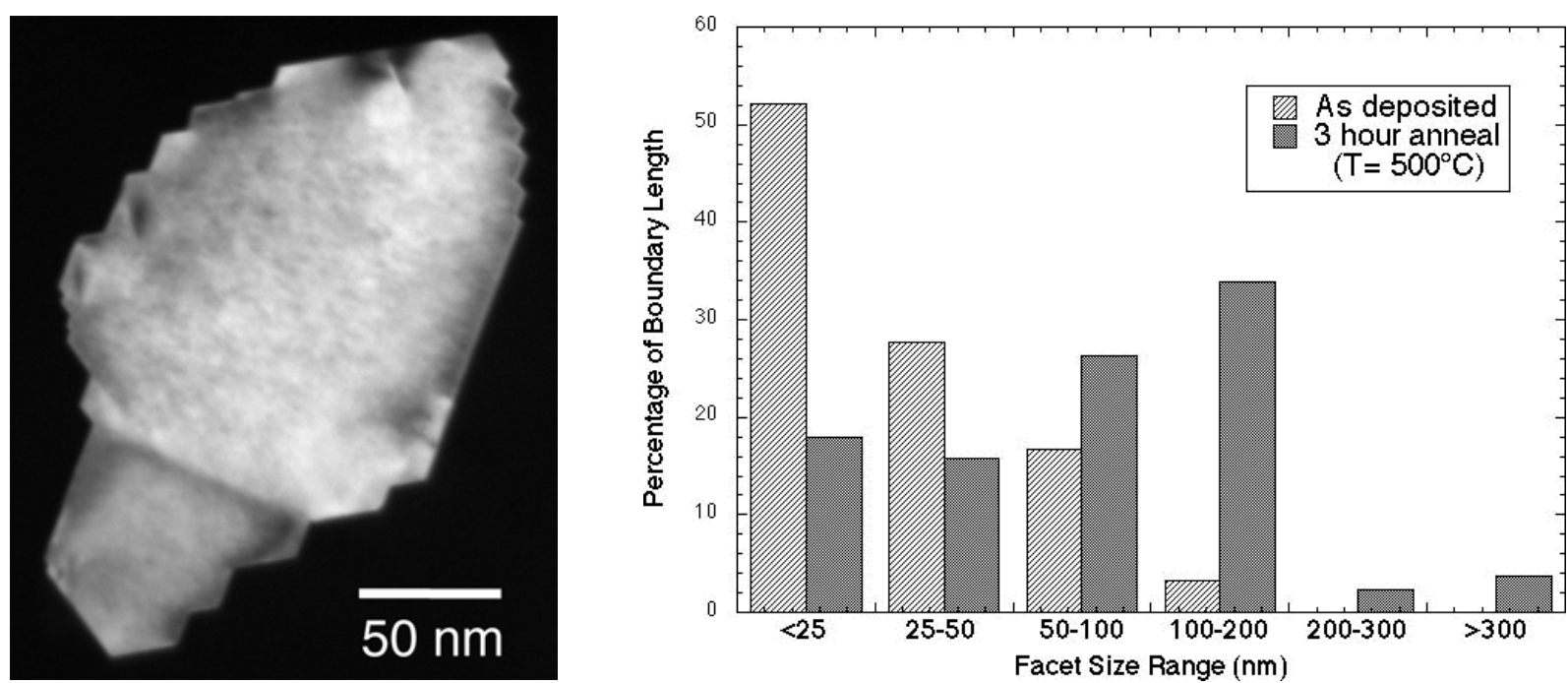

FIG. 1. (a) Darkfield micrograph showing facet steps on an enclosed grain in an as-deposited $\Sigma=3 \mathrm{Au}$ film. (b) Graph shows the proportion of total grain-boundary length as distributed across the range of facet sizes for grains before and after annealing. Prior to annealing, the majority of facets are smaller than $50 \mathrm{~nm}$. With annealing, an increased proportion of boundary length shifts to larger facet size.
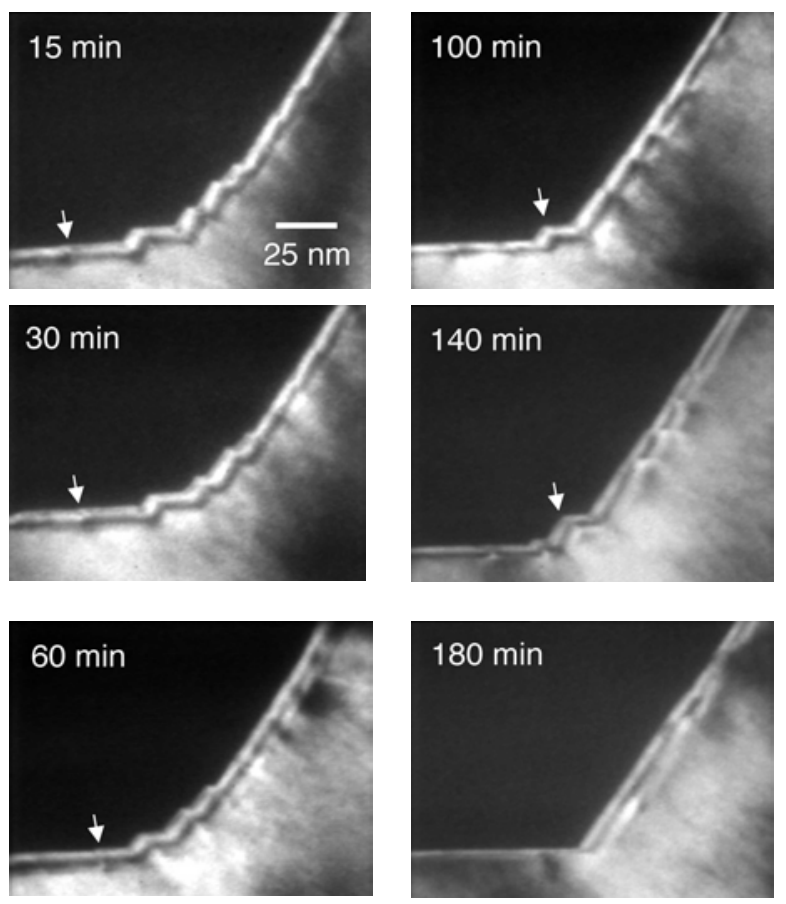

FIG 2. Stills taken from video showing motion of facets during evolution of boundary corner during annealing $\left(\mathrm{T}=490^{\circ} \mathrm{C}\right)$.

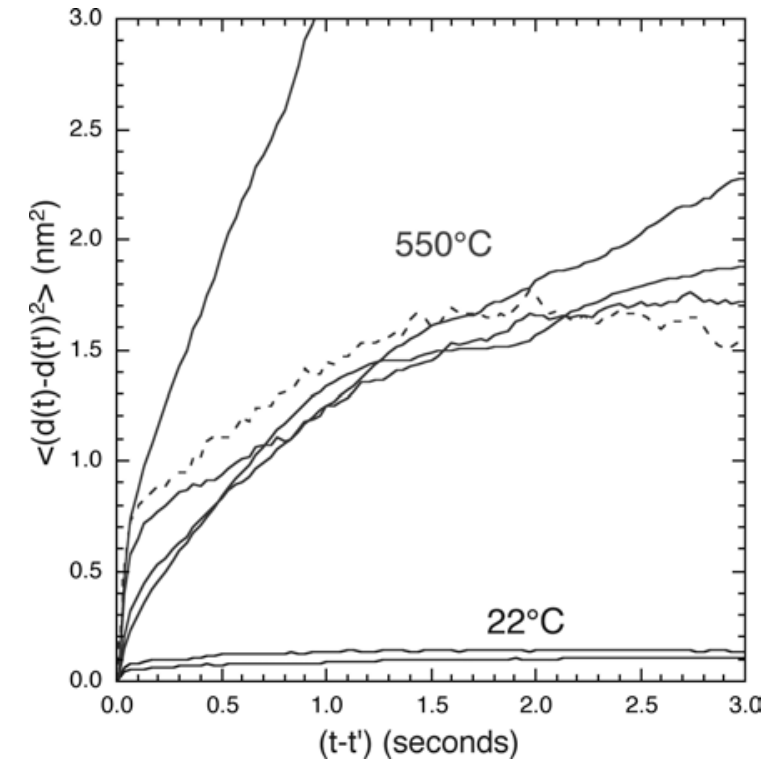

FIG 3. Thermal fluctuations in junction separation characterized by autocorrelation analysis. $\quad(\sim 1$ minute video records, sampled at $30 \mathrm{fps}$ ) Non-linearity in plot of meansquared-displacements indicates a deviation from random-walk. 